\title{
Extrinsic chirality: Tunable optically active reflectors and perfect absorbers
}

\author{
Eric Plum ${ }^{1, \text { a) }}$ \\ Optoelectronics Research Centre and Centre for Photonic Metamaterials, University of Southampton, Highfield, \\ Southampton, SO17 1BJ, UK
}

Conventional three-dimensionally (3D) chiral media can exhibit optical activity for transmitted waves, but optical activity for reflected waves is negligible. This work shows that mirror asymmetry of the experimental arrangement - extrinsic 3D chirality - leads to giant optical activity for reflected waves with fundamentally different characteristics. It is demonstrated experimentally that extrinsically 3D-chiral illumination of a lossy metasurface backed by a mirror enables tunable circular dichroism and circular birefringence as well as perfect absorption of circularly polarized waves. In contrast, such polarization phenomena vanish for conventional optically active media backed by a mirror.

Optical activity, that is rotation of the polarization state of light (circular birefringence) and differential absorption of circularly polarized waves (circular dichroism), is a well-known consequence of transmission through chiral media. It is usually observed in intrinsically 3D-chiral structures, i.e. structures that cannot be superimposed with their mirror image (Fig. 1a). Large polarization changes occur for transmission through 3Dchiral metamaterials, ${ }^{1-7}$ and natural media ${ }^{8}$ where effects can accumulate over long interaction lengths. In contrast, circular birefringence and dichroism for waves reflected from 3D-chiral solids and liquids are usually negligible. ${ }^{9-12}$

It is less well-known that optical activity also occurs in achiral structures, if the direction of the wave incident onto the material makes the experimental arrangement different from its mirror image (Fig. 1b). This is known as extrinsic 3D chirality and leads to large circular birefringence and dichroism for both transmitted ${ }^{13-17}$ and reflected ${ }^{18}$ waves as well as similar phenomena for scattering ${ }^{19}$ and second harmonic generation ${ }^{20}$ by nanostructures. Extrinsic 3D chirality is present for oblique incidence onto structured interfaces (e.g. planar metamaterials also known as metasurfaces) that lack two-fold rotational symmetry, if the structure does not have a line of mirror symmetry in the plane of incidence. Other forms of extrinsic chirality resulting in directionally asymmetric transmission ${ }^{21}$ and different photoluminescence ${ }^{22}$ for opposite circular polarizations have also been reported.

This letter demonstrates a fundamental difference between optical activity due to intrinsic and extrinsic 3D chirality. Polarization changes due to intrinsic 3D chirality will be reversed if the electromagnetic wave is reflected back through the 3D-chiral medium. In contrast, polarization changes due to extrinsic 3D chirality will not vanish under the same circumstances, providing an opportunity to tune specular optical activity and to realize perfect absorbers for circularly polarized waves.

Figure 1c,d illustrates why placing an optically active medium in front of a mirror will lead to vanishing

\footnotetext{
a) Electronic mail: erp@orc.soton.ac.uk; www.nanophotonics.org.uk
}

and enhanced optical activity in case of intrinsic and extrinsic 3D chirality, respectively. Any plane wave may be thought of as a superposition of right-handed (RCP, + ) and left-handed (LCP, -) circularly polarized components. As the reflection will reverse the wave's handedness, each circularly polarized component will interact both as RCP and as LCP wave with the intrinsically 3Dchiral medium resulting in cancelation of optical activity as both components will accumulate the same changes of amplitude and phase (Fig. 1c). In contrast, in case of extrinsic 3D chirality, the mirror reverses not only the wave's handedness, but it also changes the wave's propagation direction in a way that reverses the handedness of the extrinsically 3D-chiral light-matter interaction (Fig. 1d). Therefore, for one incident circularly polarized component, the handedness of the wave and all light-

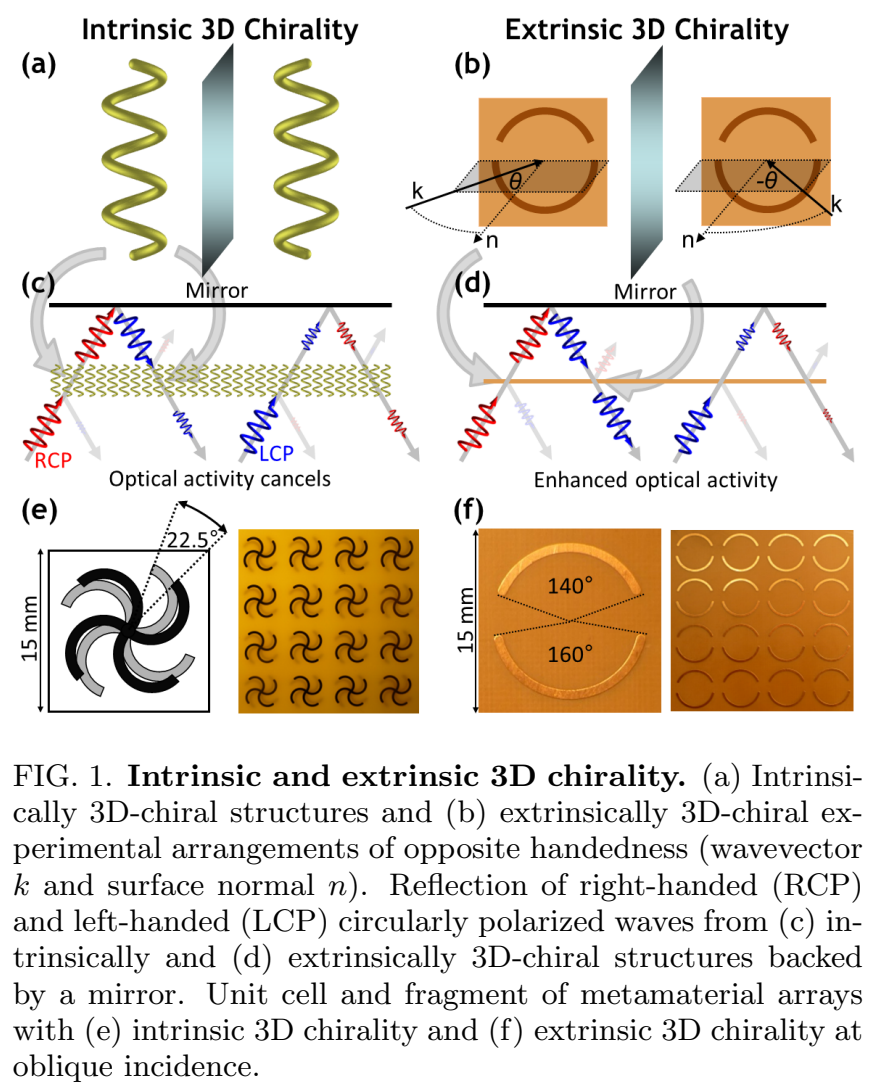



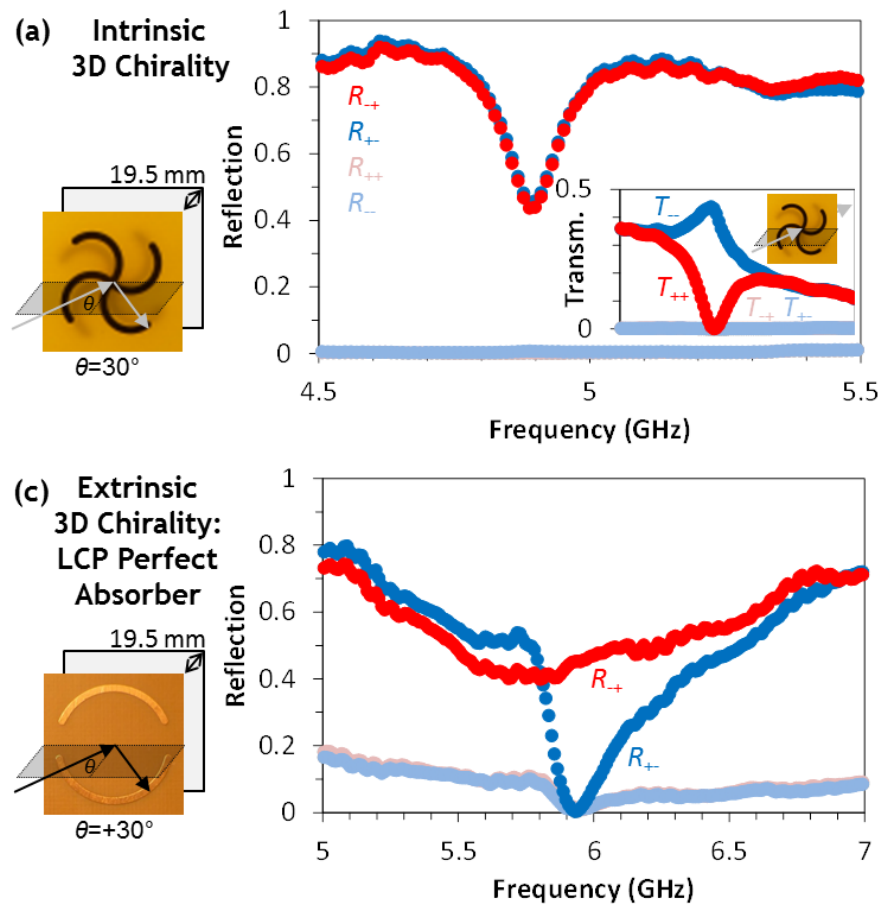
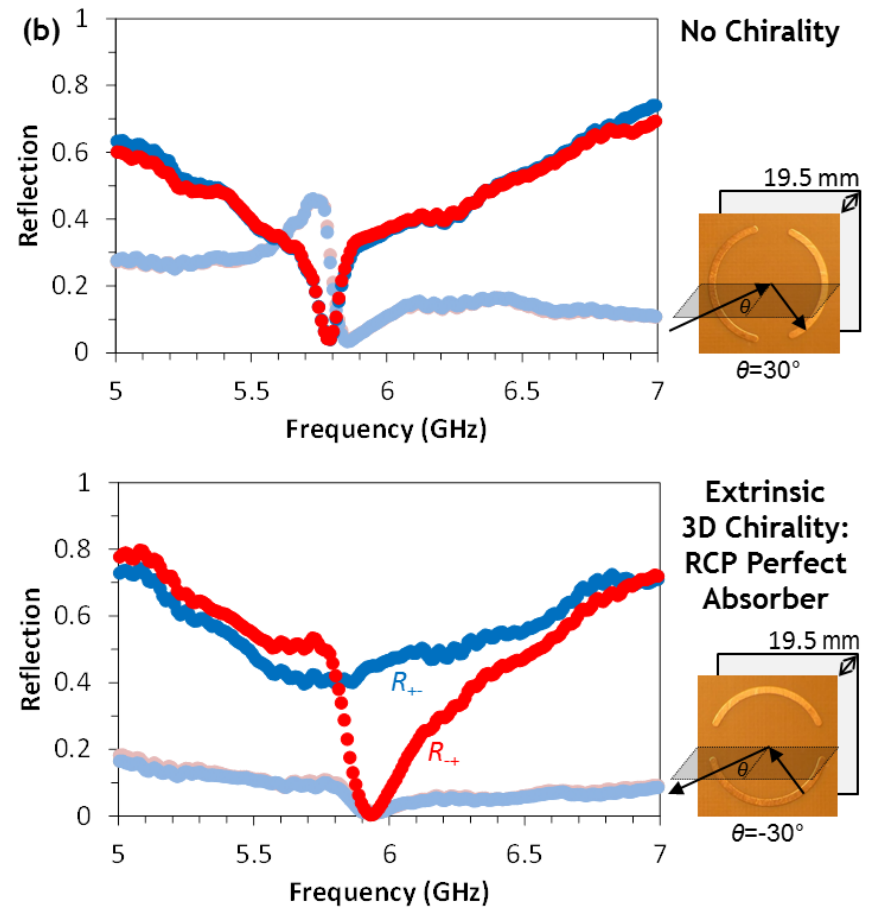

FIG. 2. Reflection spectra of achiral and chiral structures. (a) Intrinsically 3D-chiral metamaterial backed by a metallic mirror. The smaller graph shows the metamaterials's transmission without the backing mirror in the same spectral range. (b) Achiral and (c) extrinsically 3D-chiral orientations of an array of asymmetrically split rings backed by a metallic mirror. Schematics show the direction of incidence onto a unit cell of each structure with the plane of incidence marked by a gray sheet.

matter interactions will be equal, while it will always be opposite for the other incident circularly polarized component. This leads to non-vanishing optical activity, it can lead to its enhancement and it allows tuning of optical activity as changes of the metasurface-to-mirror spacing influence the relative phase and thus interference of waves (multiply) reflected by metasurface and mirror.

This study investigates the effects of intrinsic and extrinsic 3D chirality on waves reflected by a mirror in the microwave part of the spectrum using metamaterials. The intrinsically 3D-chiral metamaterial consists of mutually twisted pairs of identical metal patterns in parallel planes and it cannot become extrinsically 3D-chiral as it has two-fold (and also four-fold) rotational symmetry (Fig. 1e). Extrinsic 3D chirality was studied for an array of asymmetrically split rings which can become extrinsically 3D-chiral due to lack of two-fold rotational symmetry (Fig. 1f). It has a line of mirror symmetry and is therefore not intrinsically 3D-chiral. Both samples were made by photolithography on lossy dielectric printed circuit board substrates of $1.5 \mathrm{~mm}$ thickness. Optical activity of these structures was studied using broadband microwave antennas (Schwarzbeck BBHA 9120D) equipped with collimating lenses and a vector network analyzer (Agilent E8364B).

The effect of the metamaterial structures on electromagnetic waves can be described by complex Jones transmission $t_{i j}$ and reflection $r_{i j}$ matrices that relate the incident field $E_{j}^{\mathrm{inc}}$ to the transmitted $E_{i}^{\mathrm{t}}$ and reflected $E_{i}^{\mathrm{r}}$ fields in terms of circularly polarized components $i, j$, that is $E_{i}^{\mathrm{t}}=t_{i j} E_{j}^{\mathrm{inc}}$ and $E_{i}^{\mathrm{r}}=r_{i j} E_{j}^{\mathrm{inc}}$. The transmission and reflection coefficients in terms of intensity are given by $T_{i j}=\left|t_{i j}\right|^{2}$ and $R_{i j}=\left|r_{i j}\right|^{2}$. Circular dichroism for transmitted waves corresponds to the difference between the intensity transmission coefficients for incident circularly polarized waves of opposite handedness $\Delta T=T_{++}-T_{--}$, while circular dichroism for reflected waves is given by the difference between the reflection coefficients $\Delta R=R_{-+}-R_{+-}$. Similarly, circular birefringence for transmission and reflection corresponds to the phase differences between the field transmission and reflection coefficients, $\delta t=\arg \left(t_{--}\right)-\arg \left(t_{++}\right)$and $\delta r=\arg \left(r_{-+}\right)-\arg \left(r_{+-}\right)$. The mixed indices for reflection arise from the fact that the handedness of circularly polarized waves is reversed by a simple mirror reflection. Looking into the beam, a polarization state is defined as right-handed if the electric field vector at a fixed position is seen rotating to the right. Polarization azimuth rotation to the left is positive and the azimuth rotation due to optical activity is half of the circular birefringence.

Figure 2 illustrates the effects of intrinsic and extrinsic $3 \mathrm{D}$ chirality on waves reflected by a mirror in terms of the intensity reflection coefficients. For the intrinsically 3D-chiral metamaterial [panel (a)], optical activity in reflection $(\Delta R$ and also $\delta r)$ is zero within experimental accuracy, regardless of whether the metamaterial is backed by a mirror (as shown) or not, even though the metamaterial shows giant optical activity in transmission (see 
(a)
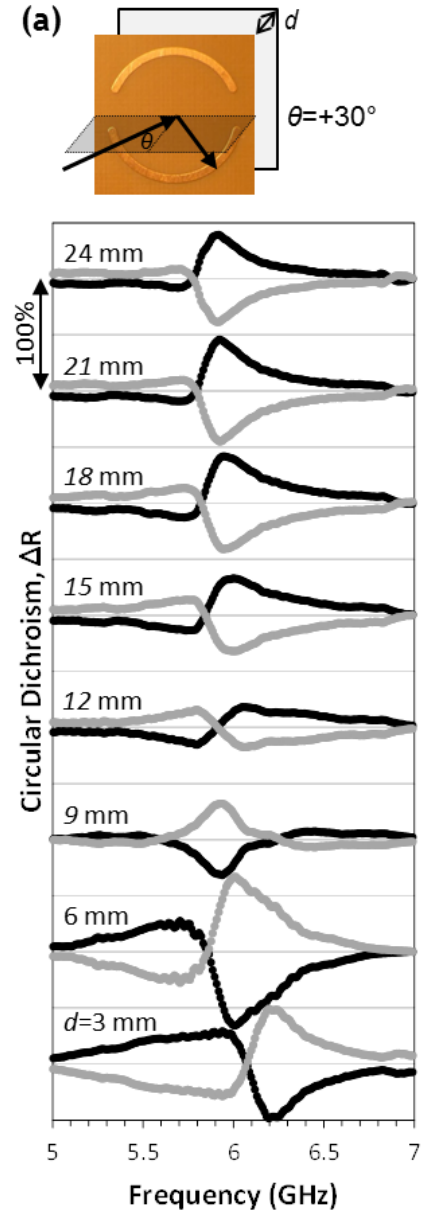

(b)
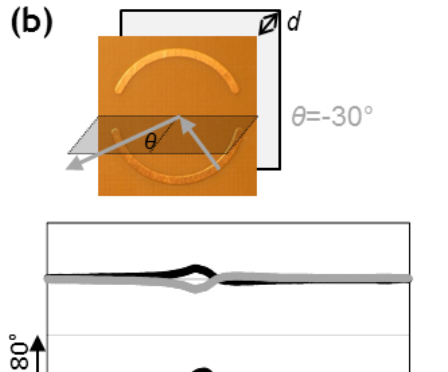

tion independent or absorb one linear polarization. ${ }^{23-30}$ The present type of perfect absorber is different as it absorbs circularly polarized waves of one handedness. This functionality cannot be realized on the basis of intrinsic $3 \mathrm{D}$ chirality, ${ }^{26}$ but recently perfect absorbers for circularly polarized waves based on intrinsic ${ }^{31}$ and extrinsic ${ }^{32}$ 2D chirality have been demonstrated and suggested, respectively. The 2D-chiral perfect absorbers preserve the handedness of the reflected circular polarization, while the extrinsically 3D-chiral perfect absorber reported here reverses it. The perfect absorber functionality of the structure studied here would not be possible without the backing mirror as absorption in a planar metasurface illuminated by one electromagnetic wave cannot exceed $50 \%$. Perfect absorption - here $99 \%$ and in principle up to $100 \%$ - becomes possible through constructive interference of coherent waves on the metasurface, ${ }^{33}$ and in the present work these coherent waves are the incident wave and the wave(s) reflected by the mirror.

Therefore, optical activity of the metasurface-mirror structure depends on the phase that waves accumulate when traveling between metasurface and mirror. This provides an opportunity to control circular dichroism and circular birefringence by changing the distance $d$ between the split rings and the mirror surface. As illustrated by Fig. 3, both circular dichroism and birefringence for reflected waves depend strongly on $d$ and both their sign and magnitude can be controlled continuously within $-66 \% \leq \Delta R \leq+66 \%$ and $-180^{\circ} \leq \delta r \leq+180^{\circ}$. Absorption $A$ reaching $99 \%$ for one incident circular polarization was observed for $d=19.5 \mathrm{~mm}$ and this case is illustrated in detail by Fig. 2c.

It is well-known that optical activity due to extrinsic 3D chirality is inherently tunable via the angle of incidence and here this is illustrated by the reversal of the effect for opposite incidence angles. However, controlling optical activity for reflected waves by changing the angle of incidence is impractical as it would dramatically alter the beam path in any application. An alternative approach where tunable optical activity is achieved through coherent control of metamaterial excitation requires two coherent phase-locked incident waves. ${ }^{34}$ In contrast, dynamic control over optical activity by placing a mirror at a variable distance from the metasurface as reported here is a practical proposition, as mirror movements on the scale of one wavelength will not affect the beam path significantly and as only a single incident wave is required.

Apart from the metasurface-to-mirror spacing, optical activity due to extrinsic 3D chirality also depends on the angle of incidence, the metasurface orientation and its unit cell's size, asymmetry and constituent materials. In particular, the chiral effects vanish when the incidence angle approaches zero, when the structure is rotated such that its line of mirror symmetry approaches the plane of incidence (Fig. 2b) and when the metasurface pattern is modified in a way that makes it two-fold rotationally symmetric, while absorption will vanish in absence of lossy materials. The spectral position of the frequency

Perfect metamaterial absorbers are usually polariza- 


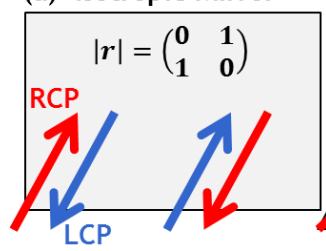

(c) Anisotropic Mirror

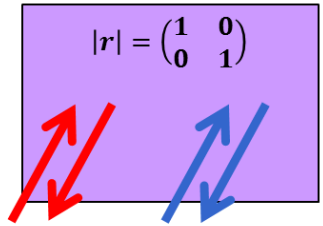

(b) $\left(\begin{array}{ll}0 & 0 \\ 1 & 0\end{array}\right)$

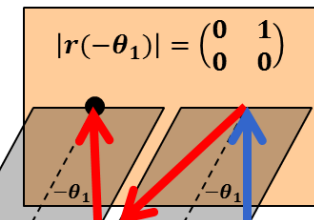

(d)

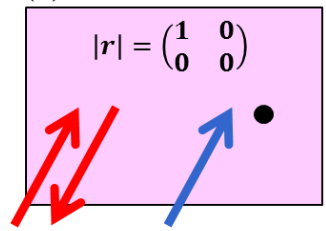

2D-Chiral Mirrors

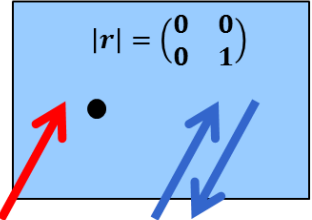

(e) Isotropic Absorber

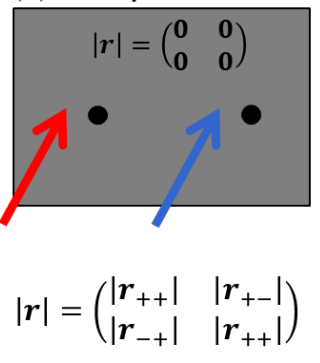

FIG. 4. Reflectors and absorbers for circularly polarized waves. (a) Normal isotropic mirrors reverse the handedness of both RCP and LCP. (b) Extrinsically 3D-chiral mirrors can reflect a single circularly polarized wave with handedness reversal, while absorbing the other. (c) Anisotropic mirrors can reflect both RCP and LCP without handedness change. (d) 2Dchiral mirrors can reflect one circular polarization without handedness change, while absorbing the other. (e) Isotropic perfect absorbers absorb both RCP and LCP.

band of optical activity is inversely proportional to the metasurface's unit cell size and its bandwidth may be expected to increase with increasing asymmetry as the effect is observed at the metamaterial's so-called "trappedmode" or Fano resonance, which becomes more broadband with increasing split ring asymmetry. ${ }^{35}$

It is interesting to compare the present work to the recent observation of specular optical activity due to extrinsic 3D chirality for a partially transparent, lossless metasurface $^{18}$. In case of the cited work, circular differential reflectance cannot be controlled with a mirror as it results from different transmission levels for RCP and LCP. In contrast, the present work reports circular dichroism for reflected waves due to differential absorption of RCP and LCP in a device that cannot transmit. In addition to being conveniently tunable and of different origin, the effects reported here (reaching $\Delta R=66 \%$, $\delta r=180^{\circ}$ and $A=99 \%$ ) are also larger than those reported in ref. $18\left(\Delta R=56 \%, \delta r=48^{\circ}\right.$ and $\left.A=0 \%\right)$.

The circular polarization perfect absorbers demonstrated here complete the family of reflectors and absorbers for circularly polarized waves. All basic functionalities illustrated by Fig. 4 can be realized by placing a metasurface in front of a conventional mirror. Handedness-inverting reflection of both RCP and LCP corresponds to the trivial case where the metasurface is not needed [panel (a)], while handedness-inverting reflection of only one circular polarization can result from extrinsic 3D chirality for metasurfaces lacking twofold rotational symmetry as shown here [panel (b)]. Handedness-preserving reflection of both circular polarizations can be achieved by using an anisotropic metasurface [panel (c)], while handedness-preserving reflection of only one circular polarization can be realized by placing a 2D-chiral metasurface in front of the mirror [panel (d)]. ${ }^{31}$ Finally, polarization-independent perfect absorption can be achieved with a mirror-backed isotropic metasurface [panel (e)]. ${ }^{27-29}$

In summary, this letter reports enhanced and tunable specular optical activity for extrinsically 3D-chiral illumination of metasurfaces when they are backed by a mirror. The effect is large and fundamentally different from conventional optical activity of intrinsically $3 \mathrm{D}$-chiral media, which is negligible in reflection. Potential applications include polarization selective detectors and reflective polarization controllers of sub-wavelength thickness including tunable polarization rotators as well as perfect absorbers and reflectors for circularly polarized waves of one handedness.

The author acknowledges fruitful discussions with Nikolay I. Zheludev and Vassili A. Fedotov. This work is supported by the Leverhulme Trust and the UK's Engineering and Physical Sciences Research Council (grants EP/G060363/1 and EP/M009122/1). The data from this paper can be obtained from the University of Southampton ePrints research repository: http://dx.doi.org/10.5258/SOTON/389763

${ }^{1}$ J. C. Bose, P. R. Soc. London 63, 146 (1898).

${ }^{2}$ E. Plum, V. A. Fedotov, A. S. Schwanecke, N. I. Zheludev, and Y. Chen, Appl. Phys. Lett. 90, 223113 (2007).

${ }^{3}$ E. Plum, J. Zhou, J. Dong, V. A. Fedotov, T. Koschny, C. M. Soukoulis, and N. I. Zheludev, Phys. Rev. B 79, 035407 (2009).

${ }^{4}$ S. Zhang, Y.-S. Park, J. Li, X. Lu, W. Zhang, and X. Zhang, Phys. Rev. Lett. 102, 023901 (2009).

${ }^{5}$ J. K. Gansel, M. Thiel, M. S. Rill, M. Decker, K. Bade, V. Saile, G. von Freymann, S. Linden, and M. Wegener, Science 325, 1513 (2009).

${ }^{6}$ M. Thiel, M. S. Rill, G. von Freymann, and M. Wegener, Adv. Mater. 21, 4680 (2009).

${ }^{7}$ Y. Zhao, M. Belkin, and A. Alù, Nat. Commun. 3, 870 (2012).

${ }^{8}$ L. Pasteur, C. R. Acad. Sci. Paris 26, 535 (1848).

${ }^{9}$ M. Silverman, N. Ritchie, G. Cushman, and B. Fisher, J. Opt. Soc. Am. A 5, 1852 (1988).

${ }^{10}$ M. P. Silverman and J. Badoz, J. Opt. Soc. Am. A 7, 1163 (1990).

${ }^{11}$ A. R. Bungay, Y. P. Svirko, and N. I. Zheludev, Phys. Rev. Lett. 70, 3039 (1993). 
${ }^{12}$ M. P. Silverman, W. Strange, J. Badoz, and I. A. Vitkin, Opt. Commun. 132, 410 (1996).

${ }^{13}$ C. W. Bunn, Chemical Crystallography (Oxford University Press, New York, 1945), p. 88.

${ }^{14}$ R. Williams, Phys. Rev. Lett. 21, 342 (1968).

${ }^{15}$ R. Williams, J. Chem. Phys. 50, 1324 (1969).

${ }^{16}$ E. Plum, V. A. Fedotov, and N. I. Zheludev, Appl. Phys. Lett. 93, 191911 (2008).

${ }^{17}$ E. Plum, V. A. Fedotov, and N. I. Zheludev, Phys. Rev. Lett. 102, 113902 (2009).

${ }^{18}$ E. Plum, V. A. Fedotov, and N. I. Zheludev, Appl. Phys. Lett. 108, 141905 (2016).

${ }^{19}$ X. Lu, J. Wu, Q. Zhu, J. Zhao, Q. Wang, L. Zhan, and W. Ni, Nanoscale 6, 14244 (2014).

${ }^{20}$ A. Belardini, M. C. Larciprete, M. Centini, E. Fazio, C. Sibilia, D. Chiappe, C. Martella, A. Toma, M. Giordano, and F. Buatier de Mongeot, Phys. Rev. Lett. 107, 257401 (2011).

${ }^{21}$ E. Plum, V. A. Fedotov, and N. I. Zheludev, J. Opt. 13, 024006 (2011).

${ }^{22}$ A. Yokoyama, M. Yoshida, A. Ishii, and Y. K. Kato, Phys. Rev. X 4, 011005 (2016).

${ }^{23}$ V. A. Fedotov, S. L. Prosvirnin, A. V. Rogacheva, and N. I. Zheludev, Appl. Phys. Lett. 88, 091119 (2006).
${ }^{24}$ V. A. Fedotov, P. L. Mladyonov, S. L. Prosvirnin, and N. I. Zheludev, Phys. Rev. E 72, 056613 (2005).

${ }^{25}$ N. I. Landy, S. Sajuyigbe, J. J. Mock, D. R. Smith, and W. J. Padilla, Phys. Rev. Lett. 100, 207402 (2008).

${ }^{26}$ B. Wang, T. Koschny, and C. M. Soukoulis, Phys. Rev. B 80, 033108 (2009).

${ }^{27}$ B. Zhang, Y. Zhao, Q. Hao, B. Kiraly, I.-C. Khoo, S. Chen, and T. J. Huang, Opt. Express 19, 15221 (2011).

${ }^{28}$ Q. Feng, M. Pu, C. Hu, and X. Luo, Opt. Lett. 37, 2133 (2012).

${ }^{29}$ G. Dayal and S. A. Ramakrishna, Opt. Express 20, 17503 (2012).

${ }^{30}$ D. Shrekenhamer, W.-C. Chen, and W. J. Padilla, Phys. Rev. Lett. 110, 177403 (2013).

${ }^{31}$ E. Plum and N. I. Zheludev, Appl. Phys. Lett. 106, 221901 (2015).

${ }^{32}$ T. Cao, C. Wei, L. Mao, and Y. Li, Sci. Rep. 4, 7442 (2014).

${ }^{33}$ J. Zhang, K. F. MacDonald, and N. I. Zheludev, Light Sci. Appl. 1, e18 (2012).

${ }^{34}$ S. A. Mousavi, E. Plum, J. Shi, and N. I. Zheludev, Sci. Rep. 5, 8977 (2015).

${ }^{35}$ V. A. Fedotov, M. Rose, S. L. Prosvirnin, N. Papasimakis, and N. I. Zheludev, Phys. Rev. Lett. 99, 147401 (2007). 